\title{
Accurate Estimation of the RMS Emittance from Single Current Amplifier Data ${ }^{1}$
}

\author{
Martin P. Stockli ${ }^{a}$, R. F. Welton ${ }^{a}$, R. Keller ${ }^{b}$, \\ A. P. Letchford ${ }^{c}$, R. W. Thomae ${ }^{b}$, and J. W. G. Thomason ${ }^{c}$ \\ ${ }^{a}$ Spallation Neutron Source, Oak Ridge National Laboratory, Oak Ridge, TN 37830, USA' \\ ${ }^{b}$ E. O. Lawrence Berkeley Laboratory, Berkeley, CA 94720, USA ${ }^{2}$
}

'ISIS, Rutherford Appleton Laboratory, Chilton, Didcot, Oxon, OXII OQX, UK

\begin{abstract}
This paper presents the SCUBEEx rms emittance analysis, a self-consistent, unbiased elliptical exclusion method, which combines traditional data-reduction methods with statistical methods to obtain accurate estimates for the rms emittance. Rather than considering individual data, the method tracks the average current density outside a well-selected, variable boundary to separate the measured beam halo from the background. The average outside current density is assumed to be part of a uniform background and not part of the particle beam. Therefore the average outside current is subtracted from the data before evaluating the rms emittance within the boundary. As the boundary area is increased, the average outside current and the inside rms emittance form plateaus when all data containing part of the particle beam are inside the boundary. These plateaus mark the smallest acceptable exclusion boundary and provide unbiased estimates for the average background and the rms emittance. Small, trendless variations within the plateaus allow for determining the uncertainties of the estimates caused by variations of the measured background outside the smallest acceptable exclusion boundary. The robustness of the method is established with complementary variations of the exclusion boundary. This paper presents a detailed comparison between traditional data reduction methods and SCUBEEx by analyzing two complementary sets of emittance data obtained with a Lawrence Berkeley National Laboratory and an ISIS $\mathrm{H}^{-}$ion source.
\end{abstract}

\footnotetext{
${ }^{1}$ This work is supported by the Office of Science, Office of Basic Energy Sciences, U.S. Department of Energy, under Contract DE-AC03-76SF-00098.

${ }^{2}$ The Spallation Neutron Source (SNS) project is a partnership of six U.S. Department of Energy Laboratories: Argonne National Laboratory, Brookhaven National Laboratory, Thomas Jefferson National Accelerator Facility, Los Alamos National Laboratory, Lawrence Berkeley National Laboratory, and Oak Ridge National Laboratory. SNS is managed by UT-Battelle, LLC, under contract DE-AC05-00OR22725 for the U.S. Department of Energy.
} 


\section{INTRODUCTION}

The emittance of a particle beam describes its suitability for focusing and transport, an important characteristic for focused charged particle beam applications. The emittance is the six-dimensional distribution of all position coordinates along the three configuration-space directions and their associated velocity coordinates, normally measured from the center of the particle distribution. It is common to reduce the emittance to three easy-to-display subsets by projecting it into the twodimensional planes $\left\{x-x^{\prime}\right\},\left\{y-y^{\prime}\right\}$, and $\left\{z-z^{\prime}\right\}$, respectively [1].

The emittance allows for the prediction of particle losses when the beam is accelerated, transported, or delivered onto a target. High-energy accelerators often encounter productivity limitations because of the radiation and activation generated by lost high-energy particles. Such particles dwell preferentially at the edge of the beam and can be removed before they gain excessive energy using carefully placed limiting apertures. This type of problem is best addressed with the size and shape of the emittance area that a certain fraction of the beam occupies in relation to the acceptance area of limiting apertures.

The emittance area, however, fails to consider the particle density distribution, which is rarely uniform across the beam, but normally peaks at the center. Accordingly, it is normally an under-proportionally small fraction of the particles that is at risk of being lost when changing the area of limiting apertures. On the other hand, the root mean square (rms) emittance takes the entire particle distribution into account and describes the focusability and transportability of a particle beam with one single, well-defined number [1-3]. Based on measured particle flux elements $c\left(x, x^{\prime}\right)$ passing through a relative position coordinate $\mathrm{x}$ with a relative velocity component $\mathrm{x}^{\prime}$, the rms emittance is defined as

$$
\begin{gathered}
\varepsilon=\sqrt{\left\langle x^{\prime 2}\right\rangle\left\langle x^{2}\right\rangle-\left\langle x x^{\prime}\right\rangle^{2}} \\
\text { with }\left\langle x^{2}\right\rangle=\frac{\sum_{a l l} x^{2} c\left(x, x^{\prime}\right)}{\sum_{\text {all }} c\left(x, x^{\prime}\right)},\left\langle x^{\prime 2}\right\rangle=\frac{\sum_{\text {all }} x^{\prime 2} c\left(x, x^{\prime}\right)}{\sum_{\text {all }} c\left(x, x^{\prime}\right)} \text {, and }\left\langle x x^{\prime}\right\rangle=\frac{\sum_{a l l} x x^{\prime} c\left(x, x^{\prime}\right)}{\sum_{\text {all }} c\left(x, x^{\prime}\right)}
\end{gathered}
$$

All terms above are normally evaluated after renormalizing $\mathrm{x}$ and $\mathrm{x}^{\prime}$ so that the first moment becomes zero, namely,

$$
\langle x\rangle=\frac{\sum_{a l l} x c\left(x, x^{\prime}\right)}{\sum_{a l l} c\left(x, x^{\prime}\right)}=0, \quad \text { and } \quad\left\langle x^{\prime}\right\rangle=\frac{\sum_{a l l} x^{\prime} c\left(x, x^{\prime}\right)}{\sum_{a l l} c\left(x, x^{\prime}\right)}=0 .
$$

The renormalization minimizes the calculated rms emittance, which corresponds to the normal process of maximizing transmission when steering a particle beam 
through a limiting aperture. The orientation and aspect ratio of the rms emittance ellipse are described by the Twiss parameters, namely,

$$
\alpha=-\frac{\left\langle x x^{\prime}\right\rangle}{\varepsilon}, \quad \beta=-\frac{\left\langle x^{2}\right\rangle}{\varepsilon}, \text { and } \gamma=-\frac{\left\langle x^{\prime 2}\right\rangle}{\varepsilon} \text {. }
$$

Many devices have been developed to measure the particle flux elements $c\left(x, x^{\prime}\right)$, such as pepper-pots, slit and collector scanners, double scanning slits, and electrical sweep scanners to name a few. All of the emittance measurement devices include a limiting entrance slit or aperture, which defines the initial position $\mathrm{x}$ of a particle flux element $\mathrm{c}(\mathrm{x})$. The particles that pass through the entrance device are intercepted farther downstream by a second device, which measures the position distribution to determine the distribution of the corresponding velocity component $x^{\prime}$ of the particles with initial position coordinate $x$. Emittance measurement hardware and associated problems and limitations have frequently been discussed in the literature [1,3-9]. All such devices have a high level of complexity and therefore require a thorough checkout and calibration, preferably with a scanning pencil beam as well as no beam, to ensure reliable and accurate emittance data.

On the data reduction side, thorough discussions seem to be limited to theoretical distributions and to the "Gaussian" analysis, for which the emittance is plotted versus the logarithm of 1 minus the enclosed beam fraction $[1,5,10]$. The Gaussian analysis gives an excellent assessment of the distribution of the beam core, roughly the inner $90 \%$ of the beam. However, it is important to include the entire beam for a proper evaluation of the rms emittance because the outer $10 \%$ contribute significantly because of the relatively high values of their position and velocity coordinates. But these values are even higher for any net background and/or artifacts measured far from the beam, which therefore need to be removed from the data or excluded from the analysis to avoid their potentially large contribution to the rms emittance estimate. This separation is intrinsically difficult and normally requires delicate judgments, which are likely to produce biased estimates with errors that are difficult to quantify. This paper focuses on the task of extracting the most accurate rms emittance estimate from emittance data. This is best accomplished with the here-introduced SCUBEEX method, a self-consistent, unbiased elliptical exclusion analysis that combines traditional rms emittance analysis methods with simple statistical methods $[11,12]$. The SCUBEEx estimates are compared with estimates obtained with traditional methods.

\section{EMITTANCE MEASUREMENTS}

For all practical purposes, emittance measurements are double slit experiments that measure the partial particle flux for broad ranges of positions $\mathrm{x}$ and corresponding

velocity components $x^{\prime}$. When the slits are in positions where they let pass a part of the beam core, the measured particle flux is a small fraction, roughly $1 \%$, of the flux 
of the entire beam. This fraction drops to the $10^{-4}$ range when measuring the beam halo, the small particle flux found outside the beam core. Going further away from the beam core, the halo gradually fades away until the measured data consist of pure background.

For this paper, we dissect the background into two components: the noise, fastvarying randomlike variations with a zero average, and the bias, the mean value, which is constant, at least locally. The bias can be zero and could vary slowly with the values of the position and velocity coordinates, although the analyses in this study are restricted to uniform biases.

Because of the gradual transition and because of the inherent noise, it is impossible to clearly distinguish between background data and data containing halo. Restricting the analysis to the core of the beam could circumvent the problem, but again would fail to yield meaningful estimates of the rms emittance.

Increasing the signal-to-noise ratio of the data can reduce the problem; for example, increasing the width of both slits increases the signal but decreases the resolution and so increases the systematic error of the rms emittance estimate $[1,5]$. Reducing the noise is a challenge, especially when the particle flux is determined by measuring the minute electrical currents, which are easily affected by electronic noise and biases. A high-quality current amplifier is essential [9] but still requires careful stability checks and careful zeroing. Equally important is an effective electromagnetic isolation of the amplifier and the current probe, which should include coaxial or tri-axial cables preferably with a low tribo-electrical dielectric. In addition, one should measure the distribution of the background in absence of beam. Subtracting the measured no-beam background data from the emittance data can eliminate the bias but increases the noise.

Current amplifiers commonly have a polarity switch to produce a positive output independent of the polarity of the input current. For this paper, we define positive as having the same polarity as the current observed when measuring the beam core, while negative refers to the opposite polarity.

In the absence of an actual current and a net bias, the current amplifier outputs background noise, small positive and negative data in a quasi-random sequence. As defined, the background noise data have a zero average and therefore do not contribute to the rms emittance because, on average, their contributions cancel each other out when weighted with the measured current $c\left(x, x^{\prime}\right)$. A positive bias, however, will cause an overestimation of the rms emittance because of the dominance of positive data, whereas a negative bias causes an underestimation of the rms emittance. Even a small bias can drastically affect the rms emittance estimate because the emittance data are normally dominated by background data and because the small bias current values are multiplied with a large range of $\mathrm{x}$ and $\mathrm{x}^{\prime}$, up to the highest values included in the emittance measurement. Accordingly, an unbiased rms emittance estimate requires bias-free data. We will show that this can be accomplished simply by subtracting a self-consistently determined bias from the emittance data.

This paper focuses on emittance measurements obtained with two slits followed by a single current probe connected to a single current amplifier; therefore, all data of a 
set can be expected to have the same bias, if any. Thorough examinations frequently reveal a small net bias even for data obtained with a perfectly zeroed amplifier, as well as for data corrected with measured background data. Electronic drifts of the amplifier, an imperfect amplifier zeroing procedure, or background biases that differ between beam and no beam, can be the cause. Such biases are normally a very small fraction of the noise amplitudes and therefore often go unnoticed.

Figure 1 shows actual emittance data as a density plot versus position $x$ and corresponding velocity component $\mathrm{x}^{\prime}$. This distribution was measured with a Penning type $\mathrm{H}$ - source operated at $-35 \mathrm{kV}$ on the ISIS ion source development rig. It is the horizontal complement to the vertical emittance data shown elsewhere in this volume [13]. The data describe an expanding beam without significant aberrations as seen in the tilted series of concentric ellipse-like areas of increasing darkness.

The figure shows both tails to be missing because the scan from -28 to $28 \mathrm{~mm}$ does not cover the full size of the beam. Tight scanning limits are often selected in a wellintended effort to save time, to optimize resolution, and/or to reduce analysis problems. Such incomplete emittance data, however, lead to underestimating the rms emittance and therefore are of questionable value. Nevertheless, such incomplete data sets are suited to demonstrating that reliable rms emittance estimates can be obtained, while being fully aware that the true rms emittance of this beam is significantly higher.

Calculating the rms emittance from all shown data yields $228 \mathrm{~mm} \cdot \mathrm{mrad}$, which is the product of the two half-axes of the rms emittance ellipse, as defined in (1) and (3). In agreement with this definition, we use $\mathrm{mm}$ mrad as unit rather than the often-used unit of $\pi \cdot \mathrm{mm} \cdot \mathrm{mrad}$, where the sometimes confusing $\pi$ is intended to be a superficial reminder that the value has to be multiplied with $\pi$ when comparing it with an emittance area.

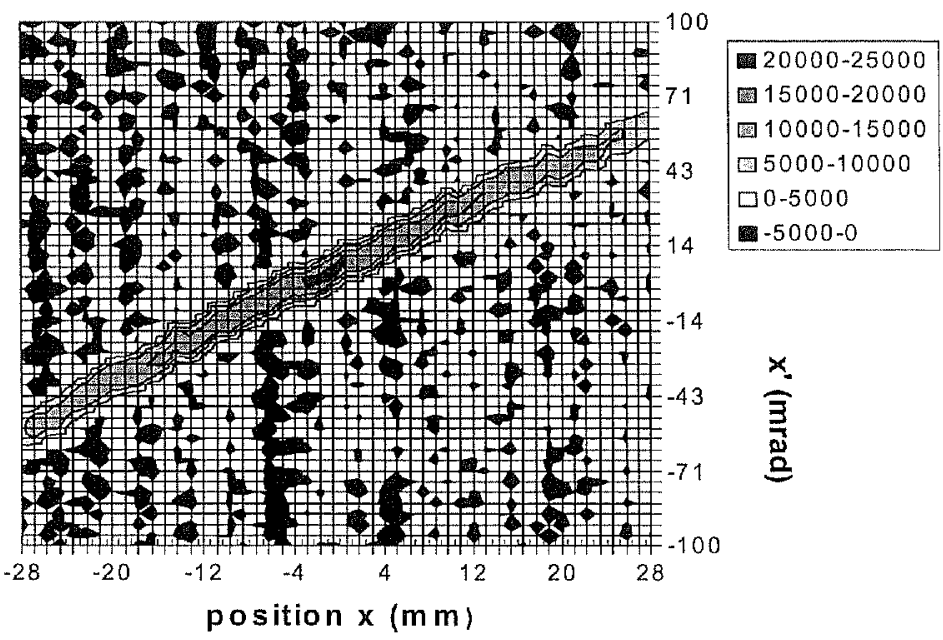

Figure 1. Emittance data from the ISIS development rig [13]. More than $85 \%$ are actual background data composed of small positive (white) and negative (black) current data. The SCUBEEx analysis uses this background to obtain unbiased, self-consistent estimates for the rms emittance and its uncertainty. The ends of the measured particle distribution are clipped causing the rms emittance to be underestimated. 


\section{HISTOGRAM ANALYSIS}

Figure 1 shows the high-current emittance data surrounded by a narrow zone of small, exclusively positive data indicated in white. Only further away from the area with higher currents can one observe negative numbers as well, indicated in black. The distribution and pattern of the data far away from the beam core appear to be compatible with a field of quasi-randomly positive and negative small numbers, as one would expect from a background with noise but without a significant bias.

A better assessment of the background data can be obtained with a histogram when plotting the number of data that fall within a small current range versus the mean current in this range. Because the background data cover only a narrow current range, they form a peak close to zero, while the data containing actual particle beam are distributed over the entire range and become visible only on the high value side where they taper off towards the highest measured current of 20,888 arbitrary units.

In emittance measurements, the currents are commonly measured in arbitrary units because any determined calibration factor cancels out when evaluating the emittance. Accordingly, we give all current values as plain numbers without any unit. Only occasionally is a current compared with the highest measured current, which then is clearly indicated by the percentage unit.

The ISIS data in Figure 2 start at -300 and have a mode close to 50 . Because this background noise is most likely symmetric or almost symmetric, it suggests that the background extends to roughly 400 with a mean about 50 . This bias value is very small, corresponding to about $15 \%$ of the highest noise amplitude, or about $0.2 \%$ of the highest measured current.

Although the histogram analysis gives a good assessment of the dominating background, it cannot isolate the distribution of the small current data measured in the beam halo because it is partly buried under the background peak.

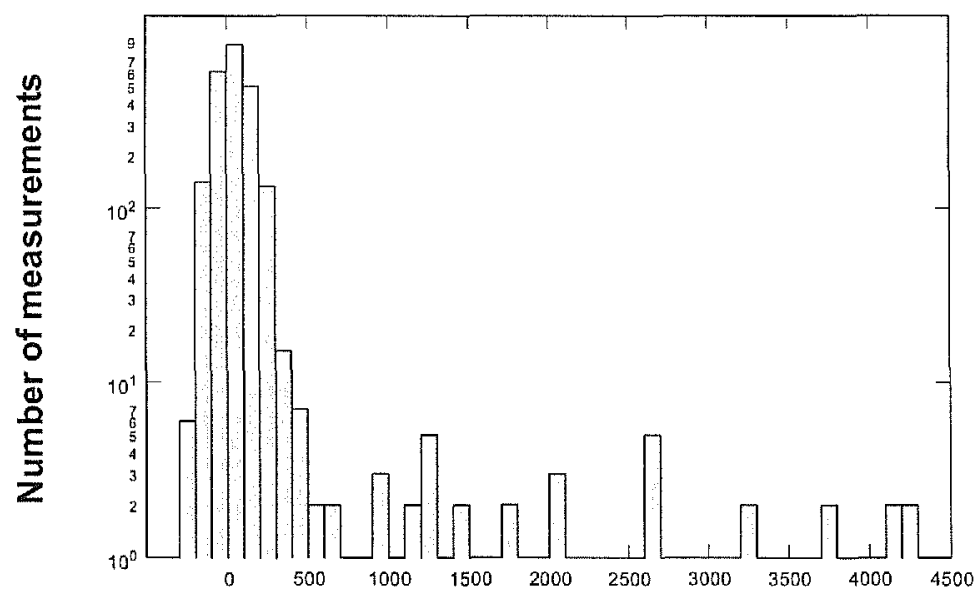

Measured current in arbitrary units

Figure 2. The histogram analysis of the ISIS emittance data shows the background data to be distributed near zero. The mode not being at zero indicates a small bias of about 50, or $0.2 \%$ of the highest measured current. 


\section{THRESHOLD ANALYSIS}

The histogram suggests that rms emittance contributions from all background can be eliminated if the threshold for the data is set at roughly 400 , meaning that all measured currents $\mathrm{c}\left(\mathrm{x}, \mathrm{x}^{\prime}\right)$ below 400 are either set to zero or excluded from all summations. To standardize this method, we quote all thresholds as a fraction of the highest measured current, clearly indicated by the percentage unit. Accordingly, the 400 correspond to a $2 \%$ threshold, which reduces the rms emittance estimate to 64.5 $\mathrm{mm} \cdot \mathrm{mrad}$.

The rms emittance estimate, however, depends on the selected threshold value. Figure 3 shows the rms emittance estimate for the ISIS data as a function of the selected threshold. Accordingly, the estimate grows rapidly when the threshold is lowered below $2 \%$ until it reaches a maximum of $270 \mathrm{~mm} \cdot \mathrm{mrad}$, with the threshold at zero where all negative values are ignored. Gradually reinstating the negative values by lowering the threshold into the negative range lowers the rms emittance estimate. It reaches the previously established $228 \mathrm{~mm}$ mrad when selecting a threshold below $-1.3 \%$, which includes all data. The rms emittance peak centered on zero is obviously the result of the positive background data not being compensated by the negative background data. The change of slope observed at $2 \%$ is sometimes used as threshold selection criterion, although it lacks a rigorous justification.

The threshold analysis and the histogram analysis sort the data according to the measured current. Because the background normally accounts for most of the data in the low current range, it dominates the results of these analyses. These dominating background features partly bury contributions from the few small, actual current measurements from the beam halo.

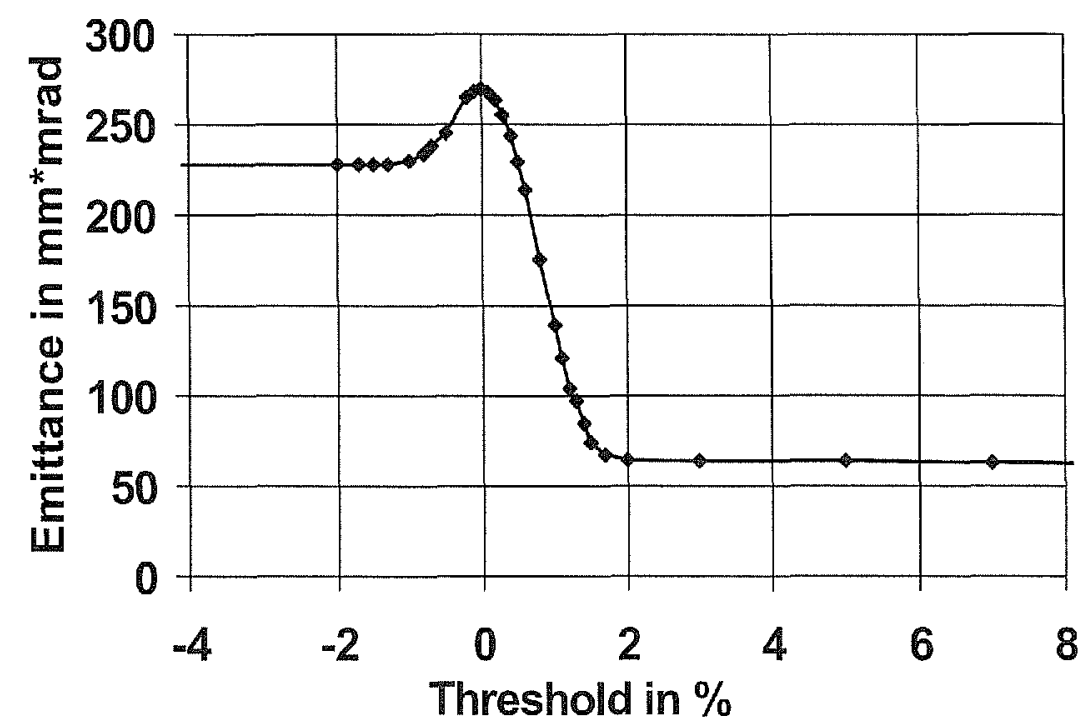

Figure 3. Threshold analysis of the ISIS emittance data shows the rms emittance estimate as a function of the threshold value expressed in a percentage of the highest measured current. The peak centered on zero is an artifact produced by excluding the negative numbers from the analysis. 


\section{ELLIPTICAL EXCLUSION ANALYSIS}

The exclusion analysis ignores data outside a certain boundary (or sets them to zero) in order to exclude all data that are assumed not to be a part of the particle beam and that are called "unphysical" by some people. This method excludes predominantly background, while giving more weight to beam-halo data because it considers their vicinity to the beam-core coordinates.

The exclusion boundary should tightly surround all data that contain actual current to maximize the exclusion of the background. Exclusion boundaries can be of various shapes, but only elliptical boundaries are used in this study because they are easy to describe and can be closely fitted to many actual phase-space distributions. The threshold for the ISIS data, for example, was held at $5 \%$ before calculating the Twiss parameters $\alpha$ and $\beta$ (3) to determine the orientation and aspect ratio of the exclusion ellipses used for Figure 4. This figure presents different quantities as a function of the half-axis-product (HAP), the product of the two half-axes of the exclusion ellipse, whether or not some part of the elliptical area falls outside the measured data field.

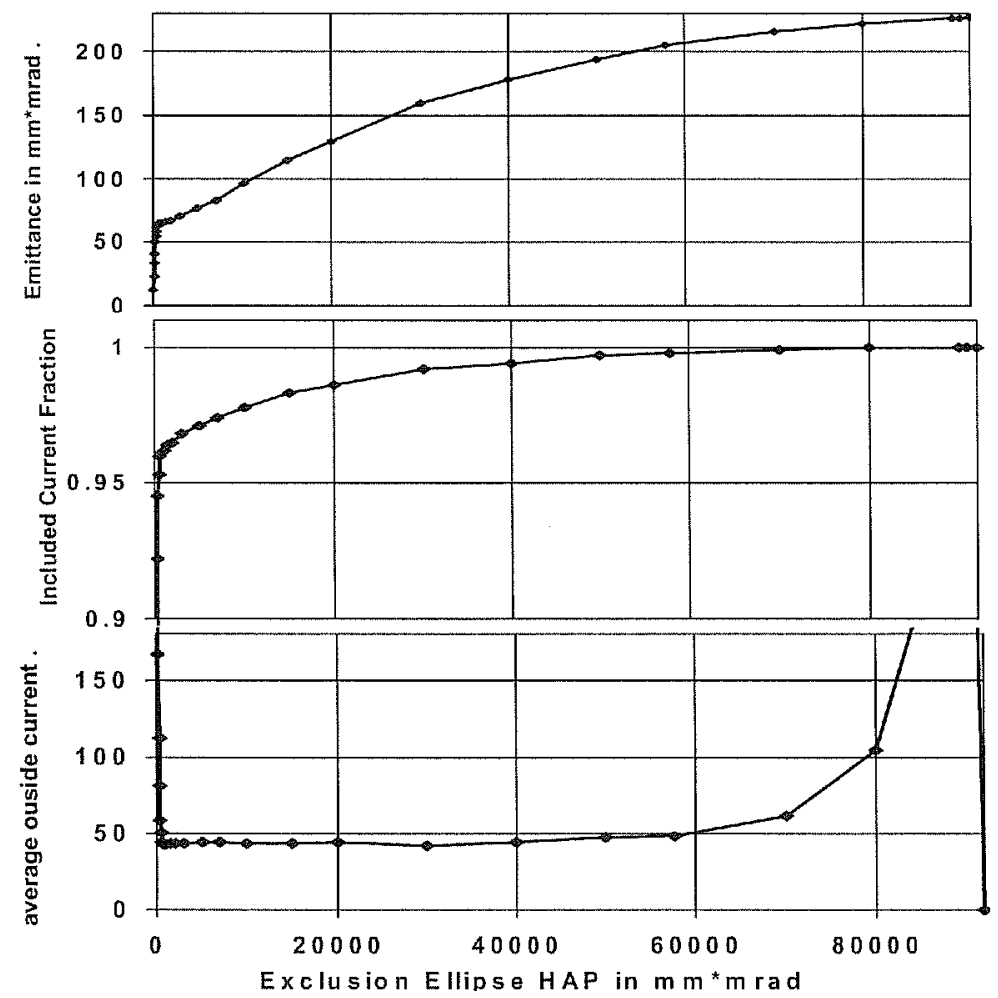

Figure 4. Exclusion analysis of the ISIS emittance data as a function of the HAP of the exclusion ellipse: (top) rms emittance estimate from the raw data within the ellipse; (center) fraction of total current within the ellipse; and (bottom) average current density outside the ellipse, which is consistent with a uniform bias current of roughly 45 in most areas. The orientation $\alpha$ and aspect ratio $\beta$ of the exclusion ellipses were determined from all emittance data exceeding $5 \%$ of the highest measured current. 
The top of Figure 4 shows the rms emittance estimate calculated from the data within the exclusion ellipse, which quickly reaches $65 \mathrm{~mm} \cdot \mathrm{mrad}$ with a HAP of 700 $\mathrm{mm} \cdot \mathrm{mrad}$, but continues to grow up to the previously established $228 \mathrm{~mm} \cdot \mathrm{mrad}$ with a HAP of $92,000 \mathrm{~mm} \cdot \mathrm{mrad}$ when the entire data field is inside the exclusion ellipse. This analysis shows that a significant part of the rms emittance growth originates from data very far from the beam core with coordinates that are physically impossible to be populated by beam particles.

The center of Figure 4 shows the sum of the currents $c\left(x, x^{\prime}\right)$ measured inside the exclusion ellipse as a fraction of the sum of the currents measured for the entire data field. The percentage of current inside a $700 \mathrm{~mm} \cdot \mathrm{mrad}$ ellipse is $96 \%$, while the $4 \%$ current outside that same ellipse accounts for a 3.5-fold increase in the rms emittance estimate.

The bottom of Figure 4 shows the current density (per data point) averaged over the excluded data outside the exclusion ellipse. The data show the average outside current to drop rapidly to 43 with HAP of $700 \mathrm{~mm} \cdot \mathrm{mrad}$ and to remain fairly constant up to $40,000 \mathrm{~mm} \cdot \mathrm{mrad}$, where it starts to increase. As the exclusion ellipse is increased, the excluded area becomes rapidly smaller because it is restricted to two remaining corners, causing drastic changes. For this reason the following analyses are restricted to exclusion ellipses with a HAP of $20,000 \mathrm{~mm}$ 'mrad or less.

Because the total of the actual particle beam current is limited, the current density of the beam halo has to decrease with increasing distance from the beam core, at least for large distances, and eventually reach zero. Accordingly, the plateau found on the bottom of Figure 4 for the average outside current measured outside $700 \mathrm{~mm} \cdot \mathrm{mrad}$ shows that these data cannot represent actual beam current but have to be background bias such as a dc-offset of the current amplifier.

Choosing an exclusion ellipse with a HAP of $700 \mathrm{~mm} \cdot \mathrm{mrad}$ results in an $\mathrm{rms}$ emittance estimate of $65 \mathrm{~mm} \cdot \mathrm{mrad}$, but this estimate clearly ignores the fact that the analysis has identified a bias in the measured currents that results in a biased rms emittance estimate.

\section{BIAS SUBTRACTION ANALYSIS}

As discussed previously, any bias, as illustrated in Figure 5a, should be subtracted from the data to obtain an unbiased rms emittance estimate. Thresholding, illustrated in Figure 5b, does not eliminate the bias from the retained part of the distribution and tends to clip the tails of the distribution. Subtraction is the only method that can
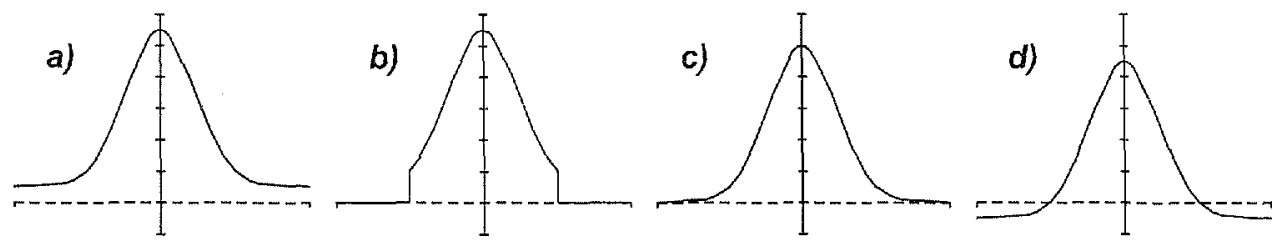

Figure 5. a) Distribution with a $10 \%$ background bias, b) after being thresholded at $20 \%$, c) after subtracting $10 \%$ bias, and d) after subtracting $20 \%$ bias. 
correctly eliminate a bias, as illustrated in Figure 5c.

However, when an excessive bias is subtracted, the method converts a significant fraction of the data to negative numbers, as illustrated in Figure $5 \mathrm{~d}$, which can lead to a gross underestimation of the rms emittance.

Figure 6 shows the rms emittance estimates of the bias-subtracted ISIS data as a function of the subtracted bias. Starting at the previously established $228 \mathrm{~mm} \cdot \mathrm{mrad}$, the rms emittance estimate drops gradually when increasing the subtracted bias. The pace, however, accelerates with increasing bias subtraction, until a subtracted bias of 48.356 estimates the rms emittance to be zero, and imaginary beyond. This underlines the potential problem of the bias subtraction: according to Figures 4 and 6 , the rms emittance estimates are imaginary if the bias is estimated from the data outside an ellipse with a HAP of $57,800 \mathrm{~mm}$ mrad. Imaginary $\mathrm{rms}$ emittance estimates are clearly unphysical and contradict the assumption that the data farthest away from the actual beam yield the most reliable bias estimates.

Accordingly, reliable bias estimates are better obtained from data in the vicinity of the particle beam but clearly outside the beam halo, a boundary that can be established with the elliptical exclusion analysis. Exclusion ellipses with a HAP between 600 and $15,000 \mathrm{~mm} \cdot \mathrm{mrad}$ estimate the bias at $44.3 \pm 1.5$, which yields an rms emittance estimates in the range between 80 and $53 \mathrm{~mm} \cdot \mathrm{mrad}$, not a very accurate estimate.

The rms emittance estimated from all bias-subtracted data is very sensitive to the subtracted bias because most data are background data (more than $85 \%$ of the ISIS data). A small error in the estimation of the subtracted bias leaves enough net bias to significantly change the rms emittance estimate. This problem can be reduced if the rms emittance is estimated from within a smaller area, which suggests combining the bias subtraction with an exclusion analysis.

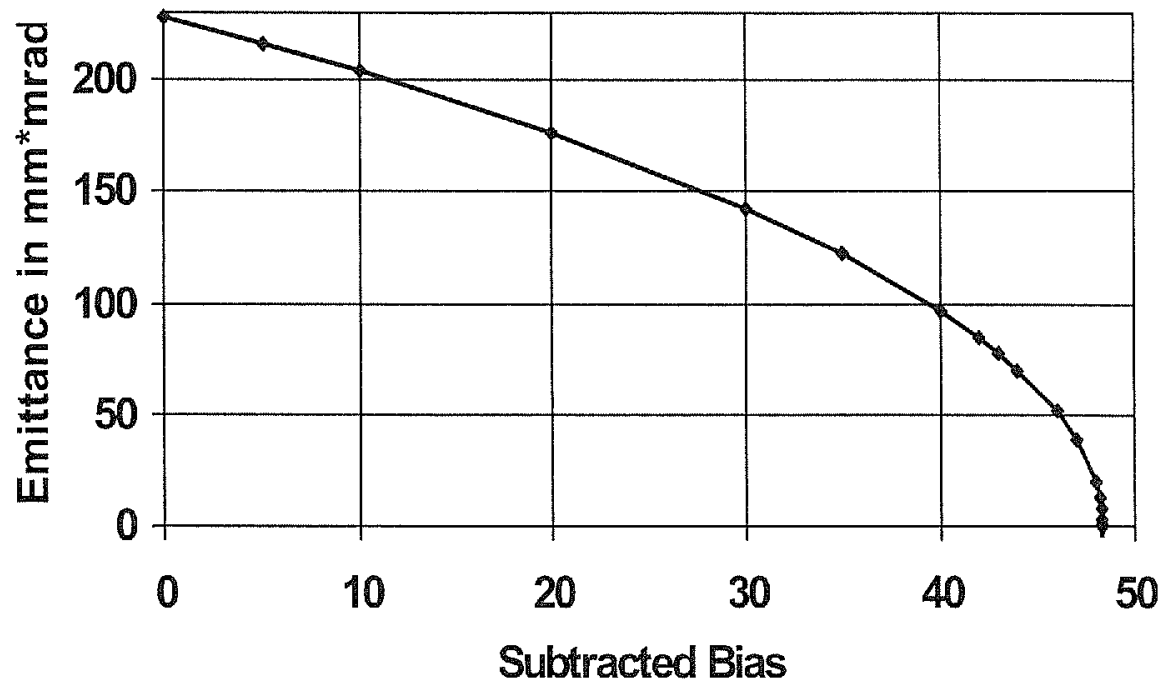

Figure 6. Bias subtraction analysis of the ISIS emittance data as a function of the subtracted bias. The dominance of background data makes the rms emittance estimate very sensitive to the subtracted bias, yielding imaginary estimates above 48.356 . 


\section{SCUBEEx, THE SELF-CONSITENT, UNBIASED ELLIPTICAL EXCLUSION ANALYSIS}

As seen previously, the elliptical exclusion analysis yields reasonable bias estimates from the average current measured outside the exclusion ellipse when the averageoutside-current versus exclusion-ellipse-HAP plot exhibits a plateau after the initial drop. The bottom of Figure 4 and the top of Figure 7 represent the same average outside currents, but the latter display is expanded by a factor of 4 and features a finer evaluation. Almost all shown average outside current estimates above $550 \mathrm{~mm} \cdot \mathrm{mrad}$ are within a range of $44.3 \pm 1.5$, showing no significant trend, but only random-like variations.

The solid line in the bottom of Figure 7 shows the rms emittance estimated from the raw data within each exclusion ellipse, which initially grows rapidly as an increasing fraction of the actual particle beam is included in the growing ellipse. However, when the ellipse HAP reaches $550 \mathrm{~mm}$ 'mrad, the rms emittance estimate starts to grow at a slower rate. The dashed line in the bottom of Figure 7 shows the rms emittance contribution from a uniform, constant current inside the exclusion ellipse, calculated from the corresponding average outside current shown in the top of the same figure.

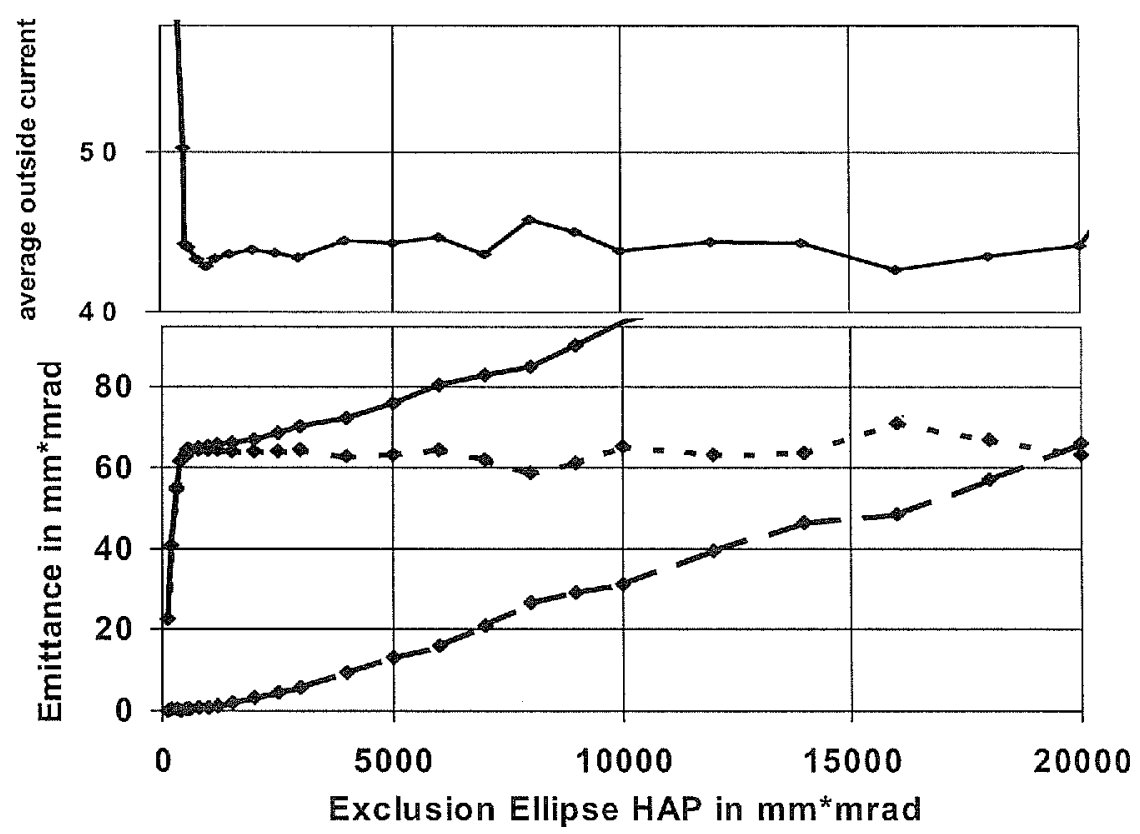

Figure 7. Unbiased exclusion analysis of the ISIS emittance data as a function of the HAP of the exclusion ellipse: top) average current density outside the ellipse (used as bias estimate) and bottom) rms emittance estimate calculated from the raw data (solid) and from the bias-subtracted data (dotted) inside the exclusion ellipse. The dashed line shows the rms emittance contribution from a uniform current equal to the estimated bias current. The rms emittance plateau reached with the unbiased data confirms reasonable bias subtraction and that all actual particle beam current was included. The ellipse parameters $\alpha$ and $\beta$ were determined from all emittance data exceeding $5 \%$ of the highest measured current. 
Starting at zero, this rms emittance contribution gains value approximately at the same rate as the rms emittance estimate based on the raw data for ellipses with a HAP in excess of $550 \mathrm{~mm} \cdot \mathrm{mrad}$. This demonstrates that the growth of the rms emittance estimate for larger ellipses is caused by bias, which can be estimated from the average outside current, and has to be subtracted from the raw data.

Rather than subtracting the estimated bias from the data and calculating the rms emittance from all data, SCUBEEx calculates the rms emittance only from the biassubtracted data within the exclusion ellipse, which reduces its sensitivity to errors in the estimated bias. For every evaluated ellipse, the SCUBEEx method first calculates the average current outside the evaluated ellipse. This average current is subtracted from all data to obtain unbiased data. The unbiased data within the ellipse are then used to calculate the unbiased rms emittance estimate. This unbiased rms emittance estimate is shown as a dotted line in the bottom of Figure 7. The estimate grows until it reaches a plateau when the exclusion ellipse exceeds a $\mathrm{HAP}$ of $550 \mathrm{~mm} \cdot \mathrm{mrad}$. The fact that the rms emittance estimate reaches a plateau is a self-consistent confirmation that the bias has been properly subtracted and that the rms emittance estimate includes the contributions from all real current measurements, even if they were buried in the noise of individual current measurements.

To form a plateau, the unbiased rms emittance estimates have to be trendless over a broad range of exclusion ellipses, as seen in Figure 7. However, the figure also shows the estimate to feature quasi-random variations. As expected, many of the noiselike variations are anticorrelated with variations of the average outside current shown in the top of the same figure. The amplitudes of these variations grow with the exclusion ellipse because of increased sensitivity to the bias estimate, a problem already discussed for the bias subtraction analysis. Therefore, future evaluations of the ISIS emittance data will be restricted to a maximum HAP of $7000 \mathrm{~mm} \cdot \mathrm{mrad}$. In the HAP range between 450 and $7000 \mathrm{~mm} \cdot \mathrm{mrad}$, all unbiased rms emittace estimates are within the range of $63.4 \pm 1.2$, an uncertainty of roughly $2 \%$.

\section{ROBUSTNESS OF THE SCUBEEx ANALYSIS}

The preceding analysis is based on varying the area of the exclusion ellipse while keeping the orientation and aspect ratio fixed. These two Twiss parameters were calculated from the data remaining after being thresholded at 5\%, a somewhat arbitrary choice. Figure 8 shows these Twiss parameters $\alpha$ and $\beta$ for the ISIS emittance data as a function of the selected threshold value. It shows $\alpha$ and $\beta$ to undergo drastic changes between -1 and $2 \%$, the range which is completely dominated by background according to the histogram analysis. Some people use the upper end of this dramatic change to locate the threshold that should exclude the entire background. The upper end at $2 \%$ seen in Figure 8 matches the change of slope observed in Figure 3, which was previously discussed as a possible selection criterion.

At higher thresholds, $\alpha$ and $\beta$ change rather slowly, making the ellipse stretch and wobble just a little, as can be seen in Figure 9. This figure shows four ellipses with a 


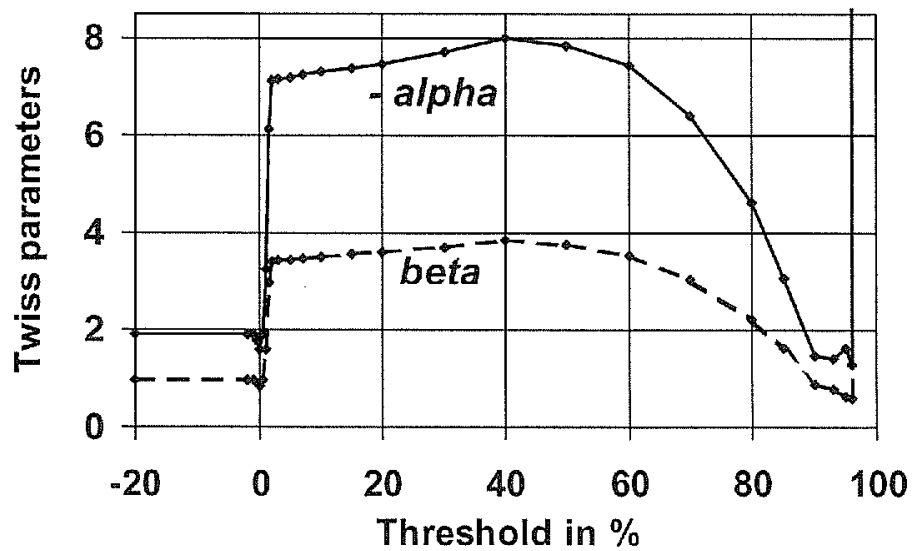

Figure 8. The threshold analysis of the ISIS emittance data shows the Twiss parameters $\alpha$ and $\beta$ as a function of the threshold. Elimination of the background produces sharp changes between -1 and $2 \%$.

HAP of $200 \mathrm{~mm} \cdot \mathrm{mrad}$ and with $\alpha$ and $\beta$ thresholds of $5 \%$ (A), $40 \%$ (B), and $80 \%$ (C). Ellipse $\mathrm{D}$ is rather roundish because its parameters were determined with a threshold below $-1.3 \%$ to include all data.

Figure 10 shows the SCUBEEx analysis repeated for exclusion boundaries scaled from the four ellipses shown in Figure 9. The dash-dotted lines are obtained by scaling ellipse $A$ and therefore are the same data as in Figure 7 except that Figure 10 features a finer evaluation and is expanded by a factor of 3 . The dashed lines, obtained by scaling the slightly elongated and twisted ellipse $B$, are practically on top of the dash-dotted lines, confirming the results obtained with ellipse A. A noticeable difference is observed from the dotted lines obtained by scaling ellipse $C$, which is less elongated. At 5000 and $5500 \mathrm{~mm} \cdot \mathrm{mrad}$, the ellipse yields unusually high bias estimates that lead to unusually low rms emittance estimates. This appears to be the same background variation that can be observed in Figure 7 with ellipse $\mathrm{A}$ at a HAP of $8000 \mathrm{~mm} \cdot \mathrm{mrad}$. If this depression is ignored, which is far from the beam, all other estimates obtained with ellipses $\mathrm{A}, \mathrm{B}$, and $\mathrm{C}$ are within the range of $63.8 \pm 1$.1. This range is in fair agreement with our previous estimate based exclusively on ellipse A, proving the robustness of the SCUBEEx method.

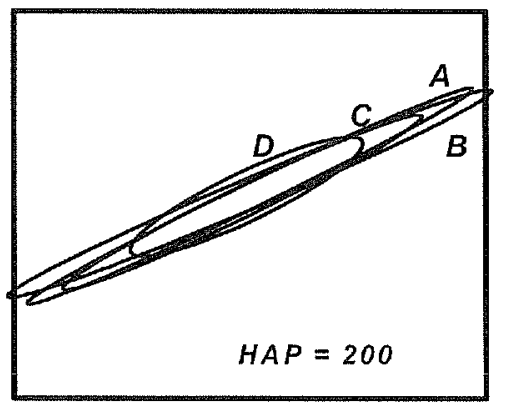

Figure 9. Ellipses with a HAP of $200 \mathrm{~mm} \cdot \mathrm{mrad}$ used for the robustness test of the unbiased elliptical exclusion analysis shown in Figure 10. Analyzing the ISIS emittance data that exceed 5\% (A), 40\% (B), or $80 \%$ (C) of the highest measured current, as well as analyzing all data (D), yielded the corresponding alpha and beta parameters. 


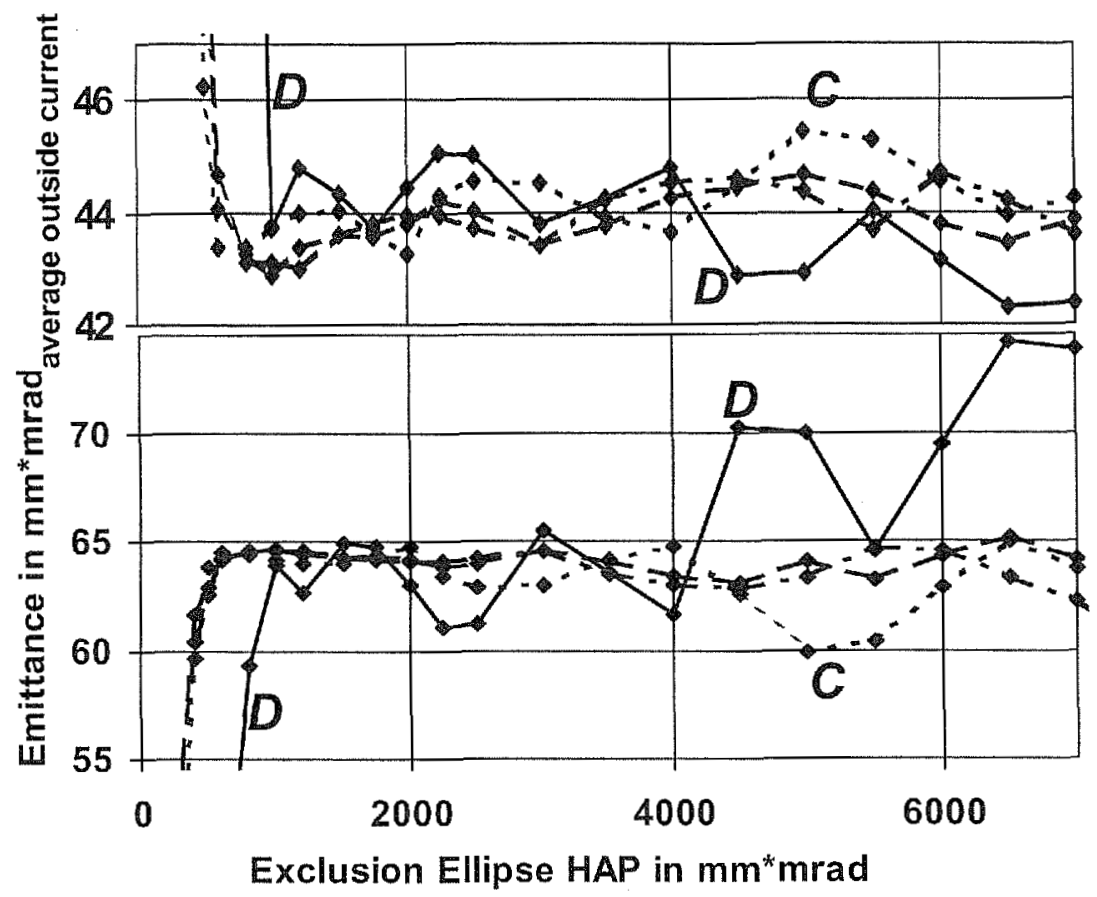

Figure 10. Unbiased exclusion analysis of the ISIS emittance data as a function of the HAP of different exclusion ellipses: top) average outside current, and bottom) rms emittance estimated from the bias-subtracted data inside the exclusion ellipses A (dash-dotted), B (dashed), C (dotted), and D (solid), shown in Figure 9. All ellipses give consistent estimates, but the variations increase in amplitude as the fit gets poorer with ellipses $C$ and $D$.

The solid lines in Figure 10 are obtained with the roundish ellipse D calculated from all data. Because of its badly fitted aspect ratio, it takes a much larger ellipse to include all actual measured current, and therefore the average outside current and the unbiased rms emittance estimate reach their plateaus with a HAP of $1000 \mathrm{~mm} \cdot \mathrm{mrad}$, almost twice the value needed for the other ellipses. For the same reason, the background variation discussed previously seems to appear at a HAP of 2400 mm'mrad, about two to three times smaller than with the other ellipses. These two effects shorten the plateau almost beyond recognition. If SCUBEEx were used exclusively with ellipse $D$, the rms emittance would probably be estimated with $63 \pm$ 2 , with a relatively low level of confidence. This example shows how the estimates benefit from selecting an exclusion boundary that tightly surrounds all emittance data that contain actual particle flux.

\section{NEGATIVE NUMBER SUPPRESSION}

According to our definition, all actual measured currents should be positive, and it is not surprising that negative current data are frequently questioned. A radical notion argues that all negative current data are unphysical and therefore ought to be 
eradicated by converting them to zero. When all negative data in emittance measurements are zeroed, their canceling benefit gets lost and the remaining positive results from the widespread background are likely to drastically inflate the rms emittance estimate. If this zeroing process is combined with an exclusion analysis, one is still likely to estimate a reasonable rms emittance because most zeroed numbers are excluded with the background and because the few, if any, zeroed numbers inside the exclusion boundary are unlikely to have a significant effect on the rms emittance estimate.

A strange situation evolves when the zeroing of negative numbers is preceded by a bias subtraction, as shown in Figure 11 for the ISIS emittance data. Starting without a bias subtraction at the previously established $270 \mathrm{~mm} \cdot \mathrm{mrad}$, the rms emittance estimate decreases rather slowly when increasing the subtracted bias. When subtracting 50, the bias estimated with Figure 2, the rms emittance is estimated at 211 mm'mrad.

Only when the subtracted bias is increased to 300 is a more reasonable estimate of $65.1 \mathrm{~mm} \cdot \mathrm{mrad}$ obtained. Around this point, the curve in Figure 11 exhibits a change of slope, which could be used as a selection criterion for this method. For higher bias subtractions, the rms emittance estimate barely changes, for example, subtracting 500 yields $63 \mathrm{~mm} \cdot \mathrm{mrad}$ and subtracting 800 yields $62 \mathrm{~mm} \cdot \mathrm{mrad}$. Even so the subtracted bias exceeds the actual bias by a huge factor, the rms emittances estimates never become imaginary because all negative numbers are suppressed. The absence of such warning bells and the insensitivity of the rms emittance estimate to the level of bias subtraction, as seen in Figure 11, gives this estimate an image of stability and accordingly a false image of credibility.

To obtain the reasonable rms emittance estimate of $65.1 \mathrm{~mm} \cdot \mathrm{mrad}$, a bias of 300 has to be subtracted, which is very close to the upper limit of the noise data, 400, which was estimated in the histogram analysis. Such a high bias subtraction is necessary because practically all background data need to be eliminated in order to eliminate their unbalanced contributions to the rms emittance estimate.

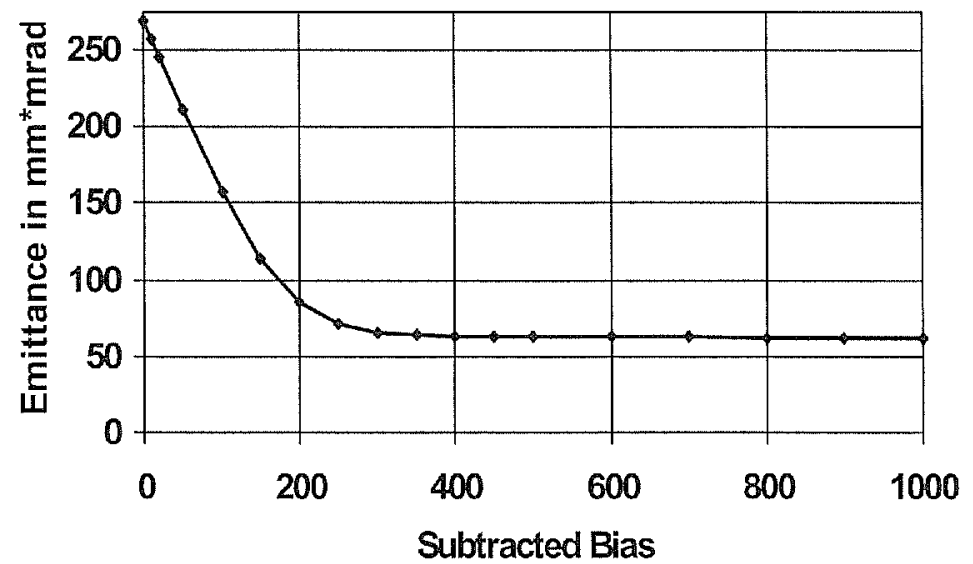

Figure 11. Bias subtraction followed by zero thresholding analysis of the ISIS emittance data as a function of the subtracted bias. Forfeiting the balancing effect of the negative current measurements, the method requires very large bias subtractions to obtain reasonable rms emittance estimates. 

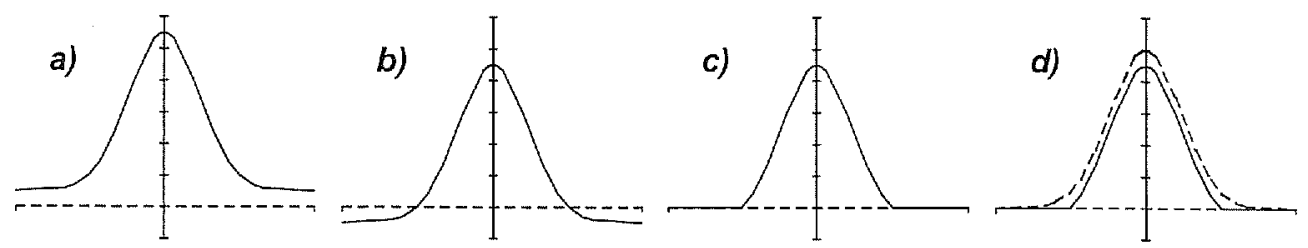

Figure 12. a) Distribution with $10 \%$ background bias, b) after subtracting $20 \%$ bias, c) after zeroing all negative numbers, and d) case c) again (solid line), in comparison with the original distribution after the proper $10 \%$ bias subtraction (dashed line).

Subtraction of an excessive bias followed by zeroing the negative numbers is illustrated in Figure 12. This figure shows how the method reduces all measured currents by the excessive bias, which is a minor problem for the high current values, but overproportionately reduces the small current values up to complete elimination. As shown in Figure 12d, this eliminates the tails of the shown distribution, and therefore, most of particle beam halo and a significant part of particle beam wings. A further increase of the subtracted bias is unlikely to yield a significant change in the rms emittance estimate because the data have practically been stripped to the core of the beam. This discussion, as well as Figure 12d, shows that this method is likely to underestimate the rms emittance up to a significant fraction.

Even though this method can give reasonable rms emittance estimates for the ISIS data, it cannot be trusted because it preferentially eliminates beam halo and wings. Negative emittance data should be a frequent occurrence when measuring with a well-zeroed current amplifier because in most positions there is no actual current and in many other positions the measured actual current is smaller than the typical noise. These negative numbers are a natural part of measuring zero or small currents in a physical world, which always features some noise, and therefore the negative numbers have to be included in any statistically sound rms emittance analysis.

\section{ANALYSIS OF THE LBNL EMITTANCE DATA}

The Lawrence Berkeley National Laboratory (LBNL) emittance data are very different from the ISIS data and are therefore a complementary test for any emittance analysis. LBNL developed a radio-frequency (rf)-driven, cesium-enhanced bucket source for SNS with a 7-mm extraction aperture, producing up to $50 \mathrm{~mA}$ of $65 \mathrm{kV} \mathrm{H}-$ [14]. The source is attached to a 10-cm-long low-energy beam-transport system (LEBT), which features two electrostatic lenses to tune the ion beam for optimal injection into the rf quadrupole (RFQ). Choosing a highly compact LEBT requires small-aperture, electrostatic lenses with inherent aberrations that cause the substantial curvature in the phase space distribution seen in Figure 13. This figure shows the same data that are shown in reference 14 as "vertical" data, with the normalized rms emittance estimated at $0.15 \mathrm{~mm}$ 'mrad after "subtracting a $1.3 \%$ background." This corresponds to $13 \mathrm{~mm} \cdot \mathrm{mrad}$ unnormalized, the standard used in this study. 


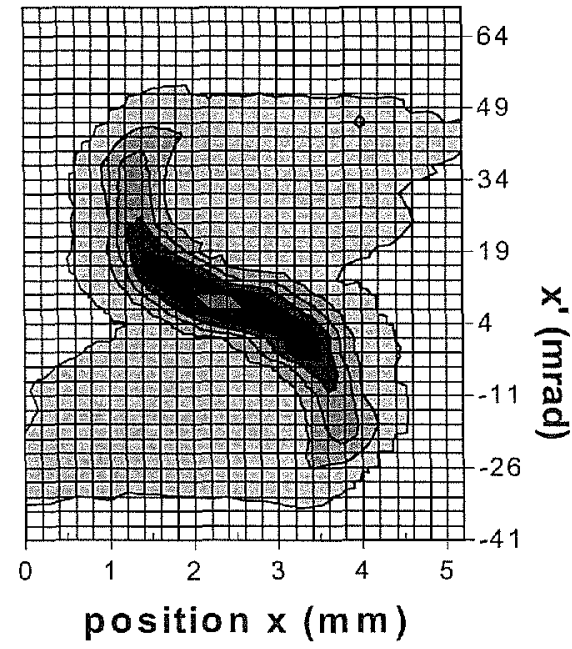

\section{$\frac{x}{3}$}

Figure 13. The measured $L B N L$ phase space distribution obtained at $33 \mathrm{~mA}$ exhibits significant curvature because of large aberrations. Unfortunately the ends of the tails were not measured.

This emittance was measured behind an 8-mm LEBT exit aperture substituting for the actual RFQ entrance aperture and therefore closely represents the emittance of the beam injected into the RFQ. The emittance data are shown in a darkening gray scale starting with white at zero, except for the intermediate gray for the highest currents between 4.1 and the peak current of 6.85. The LBNL emittance data show the core of the beam to be converging, although the low-intensity wings have components anywhere between converging and diverging.

Figure 13 shows that the extreme parts of the wings are truncated. Accordingly, a small fraction of particle beam is missing, causing an underestimation of the emittance. This figure also shows that the actual particle beam fills almost the entire data field, and therefore rather little background is included. The raw data shown in Figure 13 yield an rms emittance estimate of $16.4 \mathrm{~mm} \cdot \mathrm{mrad}$.

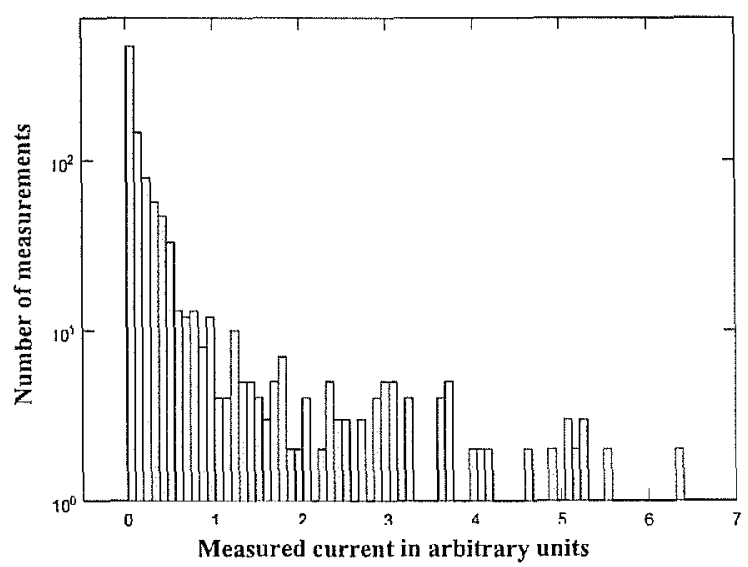

Figure 14. The histogram of the LBNL emittance data shows no negative values because the data acquisition program zeroed them. 
The histogram shown in Figure 14 reveals the absence of any negative data because they are set to zero by the data acquisition program used with the LEBT emittance device at LBNL. A closer inspection of the raw data shows that only about $40 \%$ of the background data are exactly zero, which indicates the presence of a small, positive bias. Therefore, the rms emittance must be slightly smaller than the estimate calculated from the raw data. On the other hand, the bias has to be significantly smaller than the bias estimated from the average of the background data, and therefore SCUBEEx will underestimate the rms emittance.

The histogram demonstrates how difficult it is to separate the actual small currents from the background data. The sharp drop between bins 6 and 7 could indicate the background endpoint. The corresponding $8 \%$ threshold estimates the rms emittance at $8.33 \mathrm{~mm}$ mrad. However, a closer inspection of the raw data shows that all background data are contained in the first bin. Thresholding the data at $1.3 \%$, the upper limit of the first bin, estimates the rms emittance at $15.4 \mathrm{~mm} \cdot \mathrm{mrad}$.

The threshold analysis for the Twiss parameters $\alpha$ and $\beta$ is shown in the top of Figure 15 . The $\alpha$ parameter changes significantly over the range between 0 and $15 \%$. If the upper end of this range, $15 \%$, is selected as a threshold to exclude the entire background as previously discussed, the rms emittance estimate drops to 6.71 $\mathrm{mm} \cdot \mathrm{mrad}$.

The rms emittance estimate, however, depends strongly on the selected threshold, as seen in the bottom of Figure 15. Because all negative data were zeroed, the emittance estimate remains at the highest value of $16.4 \mathrm{~mm} \cdot \mathrm{mrad}$ for negative thresholds, having lost the compensating benefit of the negative data. This figure shows a change of slope at roughly $8 \%$, which corresponds to an rms emittance estimate of $8.33 \mathrm{~mm} \cdot \mathrm{mrad}$.

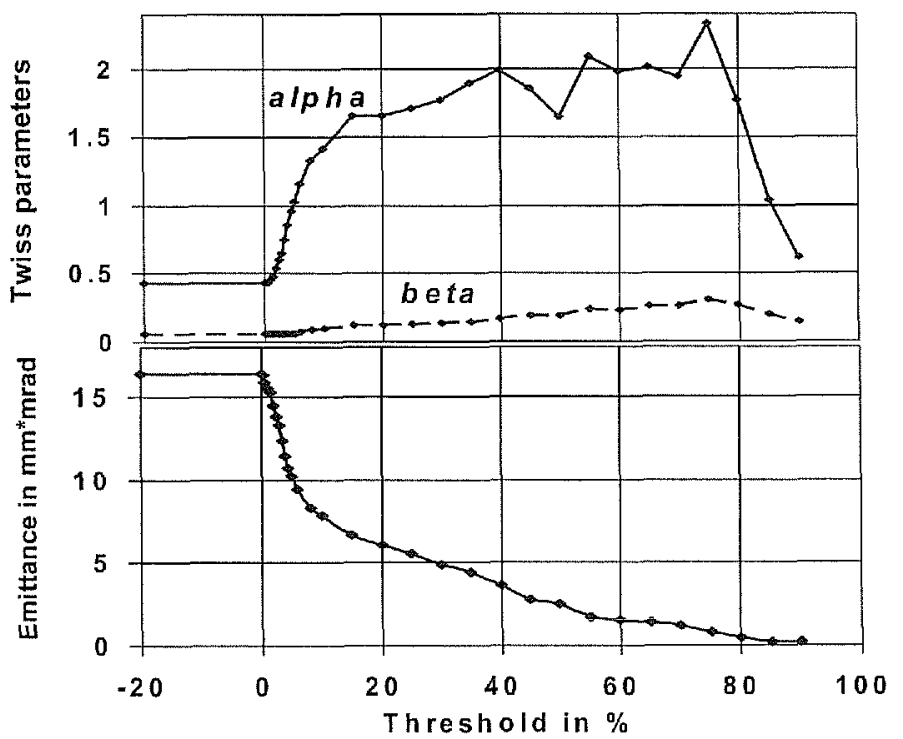

Figure 15. Threshold analysis of the LBNL emittance data shows top) the Twiss parameters alpha (solid line) and beta (dashed line) and bottom) the rms emittance estimate as a function of the threshold. 


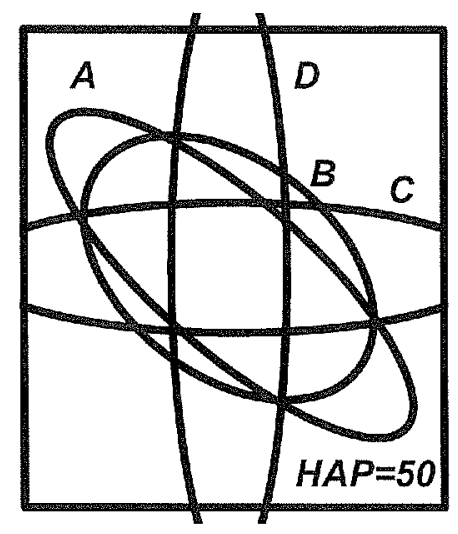

Figure 16. Ellipses with a HAP of $50 \mathrm{~mm}$ mrad used for the robustness test of the unbiased elliptical exclusion analysis shown in Figure 17. Ellipses A and B are based on Twiss parameters evaluated from thresholded data, while $\mathrm{C}$ and $\mathrm{D}$ are designed to line up with the major axes.

The wide range of rms emittance estimates obtained with different methods of selecting a threshold asks for an unbiased estimate. Figure 16 shows four ellipses, all with HAP of $50 \mathrm{~mm} \cdot \mathrm{mrad}$, which are used for the SCUBEEx analysis shown in Figure 17.

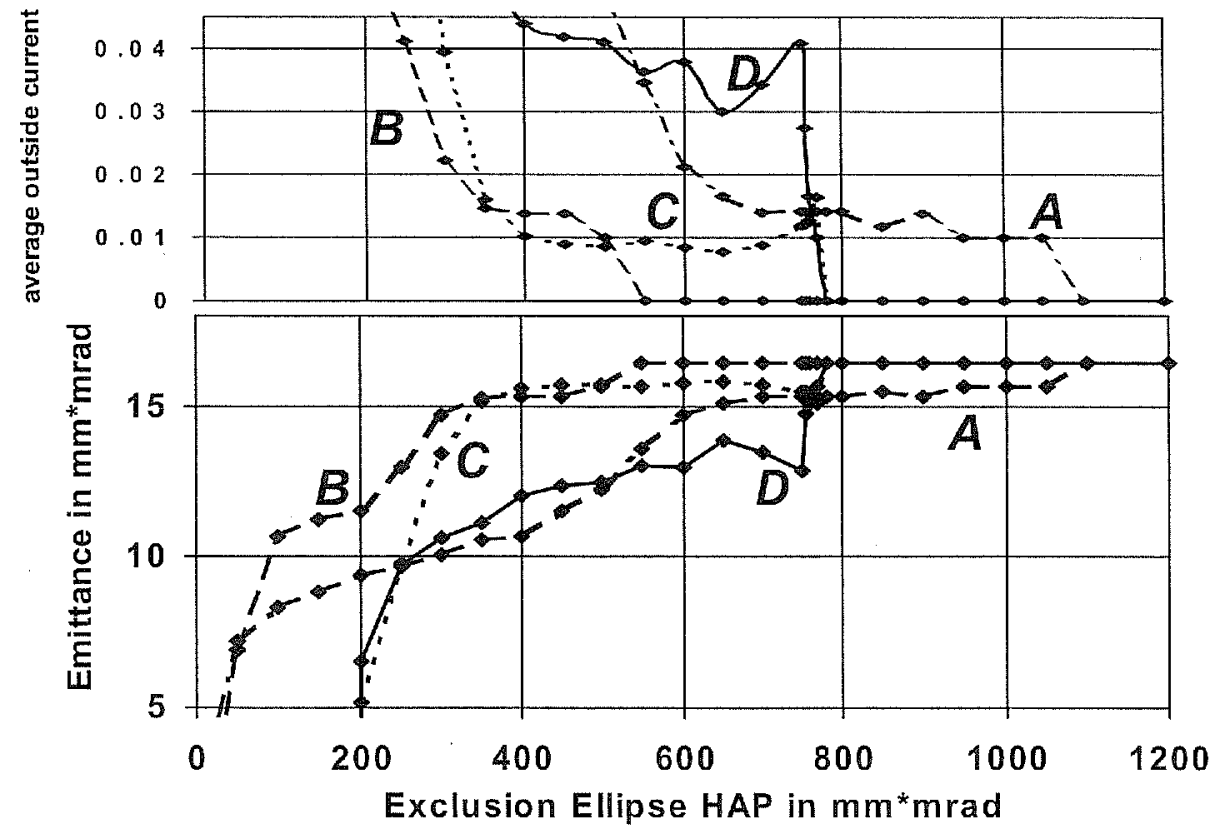

Figure 17. Unbiased exclusion analysis of the LBNL emittance data as a function of the HAP of different exclusion ellipses: top) average outside current, and bottom) rms emittance estimated from the bias-subtracted data inside the exclusion ellipses A (dash-dotted), B (dashed), C (dotted), and D (solid). The A, B, and C ellipses yield plateaus consistent with a bias of $0.011 \pm 0.003$ and an rms emittance of $15.5 \pm 0.3$. The plateaus obtained with ellipse $\mathrm{D}$ are caused by the clipped distribution tails and are therefore misleading. 
The perfectly flat $\mathrm{rms}$ emittance plateaus at $16.4 \mathrm{~mm} \cdot \mathrm{mrad}$ accompanied by zero average outside current in Figure 17 are artifacts, which are found when the exclusion ellipses include all measured data. Of relevance are the (somewhat noisy) plateaus just to the left of the trivial plateaus.

Ellipse $\mathrm{A}$ is calculated with the Twiss parameters obtained from all data exceeding $10 \%$ of the highest measured current. Accordingly, although it fits the core of the beam well, it fits the distribution wings poorly and therefore takes a relatively large HAP of $700 \mathrm{~mm} \cdot \mathrm{mrad}$ before the dash-dotted lines reach the relevant plateaus. A slightly different background bias in different comers of the data field likely causes the small change of the plateau levels at $950 \mathrm{~mm} \cdot \mathrm{mrad}$.

Ellipse B is calculated with the Twiss parameters obtained from all data and is therefore better suited to include all actual current data more rapidly with a minimum exclusion ellipse HAP of $350 \mathrm{~mm} \cdot \mathrm{mrad}$. Accordingly, the corresponding dashed lines are very similar to the dash-dotted lines from ellipse $A$ except that corresponding features are found at about half the HAP values. Unfortunately, ellipses A and B are able to isolate only the background in the upper right and lower left corners of the data field, which represents only a small fraction of all acquired background data.

Ellipse $\mathrm{C}$ was designed to simultaneously exclude the entire background on the top and bottom of the data field, and therefore the associated dotted lines feature the most pronounced plateaus.

Ellipse $\mathrm{D}$ was designed to demonstrate a potential problem: the truncated distribution wings result in plateaus, shown in solid lines, which could easily be mistaken to indicate the onset of background and thus lead to an obviously incorrect rms emittance estimate of $13 \mathrm{~mm} \cdot \mathrm{mrad}$.

The more suitable ellipses $\mathrm{A}, \mathrm{B}$, and $\mathrm{C}$ produce plateaus all consistent with a bias of $0.011 \pm 0.003$ and an rms emittance of $15.5 \pm 0.3 \mathrm{~mm} \cdot \mathrm{mrad}$, an uncertainty of only $2 \%$. This estimate differs significantly from all other estimates obtained with different thresholds selected according to different criteria.

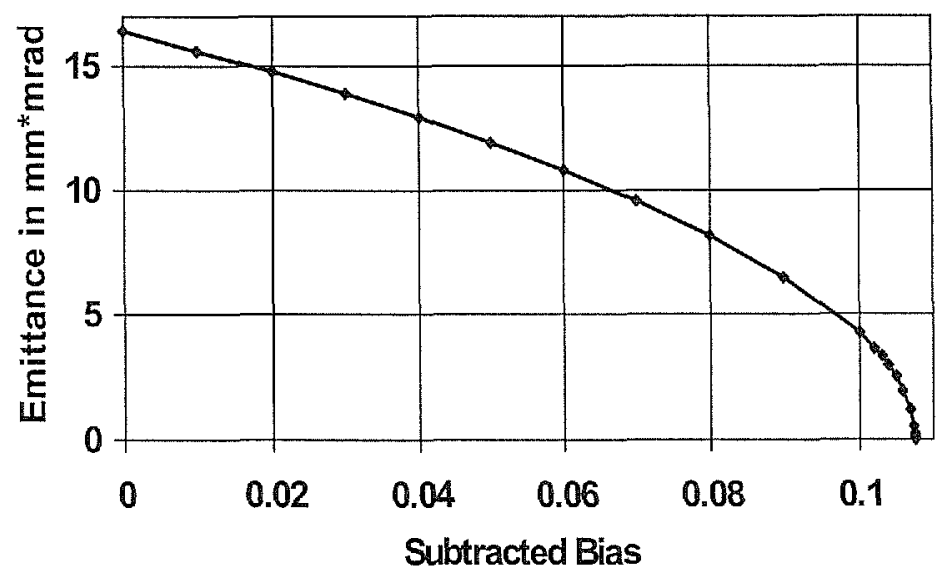

Figure 18. Bias subtraction analysis of the LBNL emittance data as a function of the subtracted bias. 
Figure 18 shows the rms emittance estimate as a function of the subtracted bias. Using the SCUBEEx bias estimate, one obtains an rms emittance estimate of $15.55 \pm$ $0.25 \mathrm{~mm} \cdot \mathrm{mrad}$, in excellent agreement with the SCUBEEx estimate.

Subtracting a bias of 0.09 (1.3\% of the highest measured current) estimates the rms emittance at $6.5 \mathrm{~mm} \cdot \mathrm{mrad}$. To obtain an imaginary emittance estimate, one has to subtract a bias of 0.10763 , which is 2.4 times larger that the highest value found in a closer inspection of the background data. The LBNL rms emittance estimates are relatively insensitive to the subtracted bias because the data occupy a rather small mm'mrad area.

Figure 19 shows the rms emittance estimate if zeroing of the negative numbers follows the bias subtraction. Using the 0.011 SCUBEEx bias estimate, one obtains an rms emittance estimate of $15.8 \mathrm{~mm} \cdot \mathrm{mrad}$. Subtracting a bias of $0.09(1.3 \%$ of the highest measured current) before clipping the negative numbers estimates the rms emittance at $13 \mathrm{~mm} \cdot \mathrm{mrad}$, obviously the method of choice in reference 14 .

The bias-subtraction-followed-by-negative-number-clipping estimate shows rather gradual changes. The most pronounced change of slope occurs between 0.2 and 0.4 , which is about 4.5 to 9 times larger than the highest value found in a closer inspection of the background data. The corresponding rms emittance estimates are 10.2 and 7.6 $\mathrm{mm} \cdot \mathrm{mrad}$, respectively. Clearly, this method yields rms emittance estimates, which scatter over a wide range. Most of these estimates severely underestimate the rms emittance, as predicted in the previous discussion.

Most methods yield very poor estimates of the LBNL rms emittance because they are unable to distinguish between the background and the small currents measured in the distribution wings and beam halo. Only SCUBEEx is able to isolate the LBNL background; therefore, only if its bias estimate is used in a bias subtraction analysis, or with the SCUBEEx method, can one obtain reliable estimates.

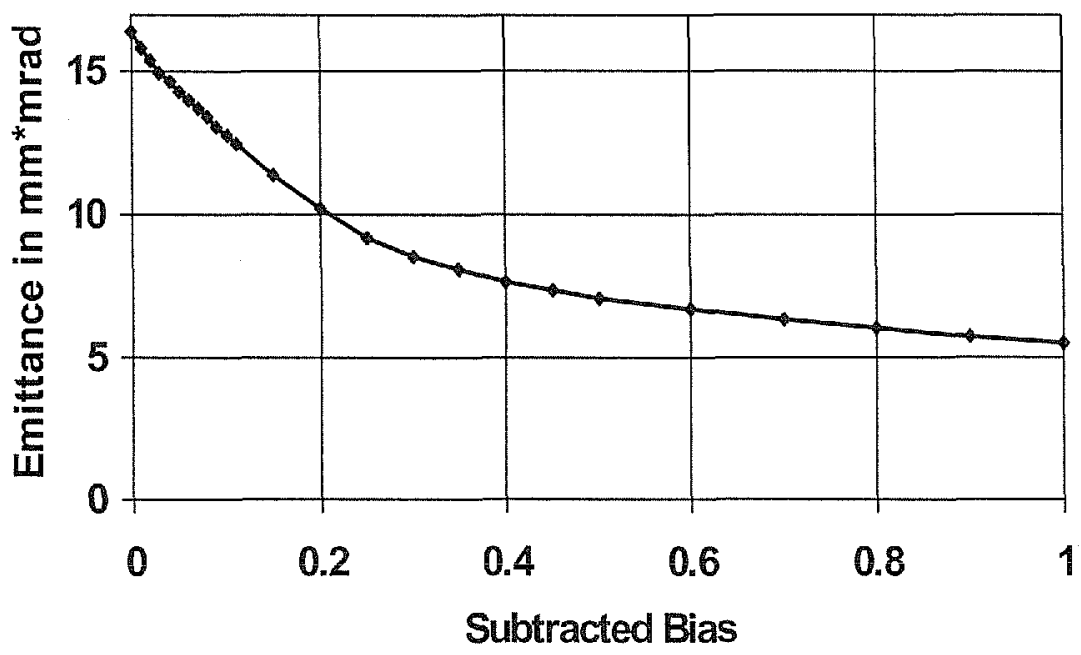

Figure 19. Bias subtraction followed by zero thresholding analysis of the LBNL emittance data as a function of the subtracted bias. 
As discussed previously, the zeroing of negative numbers by the LBNL data acquisition system causes an overestimation of the SCUBEEx bias and therefore an underestimation of the rms emittance. On the other hand, the presence of the discussed small, positive bias leads to an overestimation of the rms emittance when evaluated from the raw data. These two facts can be reconciled by estimating the LBNL rms emittance at $16 \pm 0.5 \mathrm{~mm} \cdot \mathrm{mrad}$.

\section{DISCUSSION}

The threshold and histogram analyses sort the data according to the measured currents and therefore are dominated by the background data. It is practically impossible to clearly identify features that could be caused by the much smaller fraction of data related to the small measured halo currents. Systematic threshold analyses reveal features that in some cases can be used to obtain reasonable rms emittance estimates, but it remains unclear how well such thresholds relate to the different components of the original distribution.

The exclusion analysis uses inherent information on the $\mathrm{x}$ and $\mathrm{x}^{\prime}$ coordinates of the actual distribution to separate the actual current data from the background data. It is therefore better suited to analyze the background and determine its bias, if any. Using a carefully selected exclusion boundary, one can identify small measured halo currents by comparing the average measured currents with the average measured background. However, the exclusion analysis by itself yields rms emittance estimates that depend on the actual background bias.

The SCUBEEx method estimates the bias from the data outside a carefully selected exclusion boundary before estimating the rms emittance from the bias-subtracted data within the boundary. When the exclusion area is increased, the bias as well as the rms emittance estimates normally reach plateaus, which is the self-consistent confirmation that all actual current data are inside the exclusion boundary. The uncertainty of the estimates can be estimated from the randomlike, trendless variations within the plateaus, which are caused by variations of the local average of the background data. When the exclusion area is further increased, the bias is estimated from a decreasing number of background data, and therefore the amplitudes of the observed variations increase. The increasing variations of the bias estimate cause increased variations of the rms emittance estimate. Accordingly, the evaluations of the plateaus ought to be limited to the vicinity of the beam to obtain accurate bias and rms emittance estimates.

The SCUBEEx method is somewhat robust with respect to the shape and orientation of the exclusion boundary. However, for the same aforementioned reason, the most accurate estimates are obtained when selecting the smallest possible exclusion area, which contains all data that feature actual particle beam current clearly exceeding the highest background data. For rather straight distributions, such an ellipse can be constructed using the Twiss parameters of the distribution calculated from the data thresholded above all background. Strongly curved distributions have been successfully subjected to this method as well, and the results show a surprisingly 
strong robustness. Improved credibility and accuracy can be expected when fitting the smallest exclusion ellipse that contains all data that feature actual particle beam current clearly exceeding the highest background data. A fitting procedure to accomplish this is given elsewhere [15].

Biases that vary slowly with the $\mathrm{x}$ and/or $\mathrm{x}^{\prime}$ coordinates and biases that in an isolated area significantly deviated from the average bias can lead to sloped plateaus. Such challenges can be met by estimating the bias from the average background data inside an elliptical ring surrounding the exclusion ellipse. The ring thickness needs to be optimized and actually could serve as a standard robustness test.

SCUBEEx can be used for emittance data with and without a background bias. The ISIS and LBNL data were both measured with a single current amplifier, and therefore each set was subject to only one average bias value. Interpreting the estimated bias as average bias of many amplifiers, the method has been successfully used to analyze data obtained with a multistrip detector [16]. More reliable estimates could be obtained by developing an expanded SCUBEEx method that allows for different biases for different subsets of the emittance data.

Obtaining unbiased rms emittance estimates and their uncertainty from complete data sets (including the full distribution tails) allows for fair comparisons between different ion sources [16]. The incomplete sets still allow for comparing different analysis methods. Table 1 lists the estimates obtained with easily quantifiable methods discussed previously for the ISIS and LBNL data.

The ISIS data were measured with an average bias of approximately 464 . To make the data more similar to other analyzed data, the bias was reduced by subtracting 420 from each measured value. Although this drastically reduced the rms emittance estimated from the raw data, it did not significantly change any other estimate listed in Table 1 . Consequently, both data sets have a bias of approximately $0.2 \%$ of the highest measured current, respectively. The ISIS data, however, were taken over a 19.4 times larger emittance area, containing 90\% background versus the $40 \%$ background of the smaller emittance area taken at LBNL. For these reasons, the raw

\begin{tabular}{|l|c|c|}
\hline \multicolumn{1}{|c|}{ rms emittance estimates for: } & $\begin{array}{c}\text { ISIS data } \\
\mathrm{mm} \cdot \mathrm{mrad}\end{array}$ & $\begin{array}{c}\text { LBNL data } \\
\mathrm{mm} \cdot \mathrm{mrad}\end{array}$ \\
\hline Method: & $63.8 \pm 1.1$ & $15.5 \pm 0.3$ \\
\hline SCUBEEx & 228 & 16.4 \\
\hline Raw data & $66.5 \pm 13.5$ & $15.5 \pm 0.3$ \\
Bias subtraction & $(44.3 \pm 1.5)$ & $(0.011 \pm 0.003)$ \\
\hline Bias subtraction, negative & 65.1 & 8.5 \\
numbers suppressed & $(300)$ & $(0.3)$ \\
\hline Threshold, histogram & $64.5(2 \%)$ & $8.3(8 \%)$ \\
\hline Threshold, change of slope & $64.5(2 \%)$ & $8.3(8 \%)$ \\
\hline Threshold, change of $\alpha$ & $64.5(2 \%)$ & $6.7(15 \%)$ \\
\hline
\end{tabular}

Table 1. The rms emittance estimates for the ISIS and LBNL data in mm-mrad are listed for easy quantifiable analysis methods, mostly threshold analysis. The subtracted biases and the applied threshold is shown in parenthesis. 
data overestimate the LBNL rms emittance by only $6 \%$ compared with $260 \%$ for the ISIS data.

Bias subtraction analyses yield smooth, featureless curves. But when combined with an extensive elliptical exclusion analysis to obtain self-consistent bias estimates, the bias subtraction gives credible rms emittance estimates for both cases. Small uncertainty exists for the LBNL data, but a large uncertainty for the ISIS data is the result of its much larger measured emittance area.

The ill-conceived suppression of all negative numbers preceded by bias subtraction yields smooth curves with at least one change of slope. Using the most prominent change of slope as criterion, the rms emittance estimate is credible for the ISIS data but underestimates the LBNL rms emittance by $50 \%$ because numerous low current data are mistaken as background data.

All threshold analyses give amazingly consistent and good estimates for the ISIS rms emittance, but underestimate the LBNL rms emittance by up to $57 \%$ for the same aforementioned reason.

Except for the straight evaluation from the raw data, all methods give reasonable estimates for the rms emittance of the ISIS beam. Except for the straight evaluation from the raw data, a careful bias subtraction, and SCUBEEx, all methods fail to give reasonable estimates for the rms emittance of the LBNL beam. SCUBEEx is the only method known to the authors that can unambiguously and accurately estimate the rms emittance together with credible estimates for the corresponding uncertainty for either data set. The uncertainties found to be in the few percent level for these data are much smaller than originally anticipated. Accordingly, one needs to consider additional uncertainties from other potential error sources [1].

\section{CONCLUSIONS}

Accurate and reliable rms emittance estimates require separation of the background from the real measured currents, which have to include the small currents measured in the beam halo. The common thresholding- and exclusion-analysis methods typically use a change of slope to select the optimal separation parameter. Because there is no rigorous justification, the exact selection of the separation parameter is normally based on intuition and experience, leaving some latitude for interpretation. Despite the inherent ambiguity, these methods appear to yield reasonable rms emittance estimates for well-defined data, but they appear to significantly underestimate the rms emittance of data containing numerous low current data and/or featuring a very noisy background.

The SCUBEEx method combines beneficial features of elliptical exclusion analysis and bias subtraction with simple statistical methods to obtain accurate rms emittance estimates from any measured emittance data. This method is the only one known to the authors that rigorously attempts to get unbiased estimates while simultaneously assessing their uncertainty caused mostly by variations in the background data. These rms emittance estimates include contributions from all statistically identifiable measured particle flux while excluding all contributions from statistical identifiable 
background. Of course, single parameter characterizations of phase-space distributions might not be sufficient for conducting detailed beam transport and acceleration studies. However, the unambiguous statistical identification of small particle currents and of pure background will without a doubt lead to more accurate simulations. And clearly, the improved accuracy of rms emittance estimates enables more reliable comparisons between different particle-beam-producing systems.

\section{ACKNOWLEDGMENTS}

This detailed study of rms emittance analysis has benefited significantly from discussions with our colleagues Alexander Aleksandrov, Reinard Becker, Stuart D. Henderson, Norbert Holtkamp, Miguel Olivo, Dave Olson, Ben A. Prichard, Jr., Paul Schmor, Ken Reece, Joseph D. Sherman, John W. Staples, James E. Stovall, Eugene P. Tanke, and Marion M. White. Special thanks go to Rudy Damm and Paul Gibson for providing the relief from other duties needed to make this work possible.

\section{REFERENCES}

1. LeJeune, C., and Aubert, J., in Applied Charged Particle Optics, edited by A. Septier, Academic Press, New York, 1980, pp. 159-259.

2. Keller, R., in The Physics and Technology of Ion Sources, edited by I. G. Brown, John Wiley and Sons, New York, 1989 , pp. 23-52.

3. Zhang, H., in Ion Sources, Springer, Berlin, 1999, pp. 58-65 \& 432-446.

4. Strehl, P., in Handbook of Ion Sources, edited by B. Wolf, CRC Press, New York, 1995, pp. 385-452.

5. Allison, P., in Production and Neutralization of Negative Ions and Beams, edited by J. Alessi, American Institute of Physics, New York, 1987, pp. 465-481.

6. Alessi, J, in Production and Neutralization of Negative Ions and Beams, edited by A. Hershcovitch, American Institute of Physics, New York, 1990, pp. 526-533.

7. Yuan, D., Jayamanna, K., Kuo, T., McDonald, M., and Schmor, P., Rev. Sci. Instrum. 67, 1275-1276 (1996).

8. Hamabe, M., Kuroda, T., Sasao, M., Nishiura, M., Wada, and M., Gularay, S.K., Rev. Sci. Instrum. 71, 1104-1106 (2000).

9. Dietrich, J., Mohos, I., Häuser, J., Riehl, G., and Weander, F., Proceedings of the $8^{\text {th }}$ European Particle Accelerator Conference, 2002, pp. 1864-1866.

10. Holmes, A. J. T., in The Physics and Technology of Ion Sources, edited by Brown, John Wiley and Sons, New York, 1989, pp. 53-106.

11. Eadie, W.T., Drijard, D., James, F.E., Roos, M., and Sadoulet, B., Statistical methods in Experimental Physics, North-Holland, Amsterdam, 1971.

12. Rabinovich, S., Measurement Errors, Theory and Practice, American Institute of Physics, New York, 1995.

13. Thomason, J. W. G, in these proceedings.

14. Keller, R., Thomae, R., Stockli, M., and Welton, R., in these proceedings.

15. Keller, R., Sherman, J.D., and Allison, P., IEEE Trans. Nucl. Sci. 32, 2579-2581 (1985).

16. Welton, R. F., Stockli, M.P., Keller, R., Thomae, R.W., Thomason, J., Sherman, J., and Alessi, $J$, in these proceedings. 